\title{
An Acetylcholinesterase-Mediated Density Shift Technique Demonstrates that Coated Vesicles from Chick Myotubes May Contain Both Newly Synthesized Acetylcholinesterase and Acetylcholine Receptors
}

\author{
Kathleen Porter-Jordan, ${ }^{*}$ Robin J. J. Benson, $\uparrow$ Patricia Buoniconti, ${ }^{*}$ and Richard E. Fine ${ }^{\star} \neq$ \\ Departments of *Biochemistry, †Anatomy, and ¥Physiology, Boston University School of Medicine, Boston, \\ Massachusetts 02118
}

\begin{abstract}
Coated vesicles isolated from 17 d chick embryo skeletal muscle contain acetylcholine receptors (AChRs) as shown by the presence of specific, latent binding sites for ${ }^{125}$ I-alpha bungarotoxin ( $\left.{ }^{125} \mathrm{I}-\alpha-\mathrm{BTX}\right)$. Since these coated vesicles also contain $\mathrm{AChE}$ (Benson et al., 1985), we hypothesized that a coated resicle could carry both molecules: one an integral membrane protein, the other a secreted protein. An AChE-mediated density shift technique was used to obtain data that indicate that most isolated coated vesicles contain $\mathrm{AChE}$ and that some contain AChRs as well. Similar results were obtained with coated vesicles isolated from cultured chick embryo myotubes treated briefly with diisopropylfluorophosphate (DFP) to inactivate all preexisting $A C h E$ and allowed to synthesize AChE for $2^{1 / 2}$ hr. These data are compatible with the hypothesis that both an integral plasma membrane protein, $\mathrm{AChR}$, and a secretory protein, $\mathrm{AChE}$, traverse the identical pathway after synthesis, as proposed by Rotundo and Fambrough (1980a). We suggest that coated vesicles are important intermediates in the exocytotic pathway, and that the large percentage of coated vesicles utilized for exocytotic transport can explain the rapid net increase in surface area achieved during myotube development. We also discuss the potential utility of the AChE-mediated density shift in studying the exocytotic and endocytotic pathways in other cell types, and possible pitfalls associated with its use.
\end{abstract}

The majority of AChE synthesized by embryonic chick skeletal muscle is destined for secretion (Rotundo and Fambrough, $1980 \mathrm{~b})$. We have demonstrated that isolated coated vesicles from embryonic muscle contain newly synthesized, soluble $\mathrm{AChE}$ (Benson et al., 1985). There is evidence that AChE and the acetylcholine receptor ( $\mathrm{AChR}$ ), an integral plasma membrane glycoprotein also synthesized by chick embryonic muscle cells, follow the same intracellular pathway from the rough endoplasmic reticulum to the Golgi apparatus en route to the plasma membrane (Rotundo and Fambrough, 1980a). This evidence indicates that both proteins require approximately $3 \mathrm{hr}$ after synthesis to reach the cell surface and that inhibitors of exocytotic protein transport, including monensin and nigericin, equally inhibit the transfer of both proteins to the cell surface. It has therefore been suggested that there may be a common transport vehicle for both.

Received Dec. 6, 1985; revised Mar. 17, 1986; accepted Apr. 24, 1986.

We wish to thank Michaelle Montgomery for expert secretarial assistance. This research was supported by Grant R01 NS 10582 from the National Institutes of Health.

Correspondence should be addressed to Dr. Robin J. J. Benson, Dept. of Biochemistry K-406, Boston University School of Medicine, 80 E. Concord St., Boston, MA 02118.

Copyright (C) 1986 Society for Neuroscience 0270-6474/86/113112-08\$02.00/0
In this paper, we present evidence that coated vesicles isolated from both chick embryo skeletal muscle and cultured myotubes contain AChRs as indicated by specific, latent binding of ${ }^{125} \mathrm{I}-$ alpha bungarotoxin ( ${ }^{125}$ I- $\alpha$-BTX). A recent report by Bursztajn and Fischbach (1984) demonstrates the presence of AChR-containing coated vesicles in cultured embryonic chick skeletal muscle cells by histochemical methods.

We also describe here a novel AChE-mediated density shift technique which allows us to determine that a single coated vesicle may carry both newly synthesized AChE and AChR molecules. Quantitative information obtained from this technique suggests that essentially all $\mathrm{AChR}$ molecules are contained in coated vesicles that contain $\mathrm{AChE}$ as well. We also find that over $75 \%$ of isolated coated vesicles contain AChE molecules. These results are consistent with the hypothesis that both proteins are transported along the exocytotic pathway via the same vesicle(s), and implicate the coated vesicle as the vesicle type employed.

A preliminary report of these results has been published (Porter-Jordan et al., 1982).

\section{Materials and Methods}

Coated vesicle purification from whole muscles and cultured nyotubes

Coated vesicles were purified from either $17 \mathrm{~d}$ chick embryo skeletal muscle or from cultured chick embryo skeletal myotubes that had been treated with diisopropylfluorophosphate (DFP) and allowed to synthesize new AChE for $2^{1 / 2} \mathrm{hr}$ by the methods described by Benson et al. (1985). For the experiments designed to demonstrate specific ${ }^{125} \mathrm{I}-\alpha$ BTX binding to coated vesicle-associated AChRs, we employed coated vesicles purified from sucrose density gradients. For the experiments involved in density shifting AChE-containing coated vesicles, sucrose gradient-purified coated vesicles were subjected to an additional sedimentation through a Ficoll- $\mathrm{D}_{2} \mathrm{O}$ gradient that removes aggregated coated vesicles and filaments as described by Benson et al. (1985). Protein determinations were made by the fluorescamine method (Sims and Carnegie, 1975), the Bradford (1976) method, or by the method of Lowry et al. (1951). AChE assay was performed using the method of Johnson and Russell (1975).

\section{Bungarotoxin binding assay on membrane fractions and} coated vesicles

Coated vesicles or membrane fractions, 30-50 $\mu \mathrm{g}$, resuspended in 0.1 м 2-( $N$-morpholino-)ethane sulfonic acid, $1 \mathrm{mM}$ EGTA, $0.5 \mathrm{~mm} \mathrm{MgCl} 2$, and $0.02 \% \mathrm{NaN}_{3}$, pH 6.5 (MES buffer), were incubated in $90 \mu \mathrm{l}$ MES buffer containing $0.01 \%$ ribonuclease and $0.125 \% \mathrm{BSA}$, with or without $0.025 \%$ taurodeoxycholate (tDOC) to permeabilize membranes for at least $2 \mathrm{hr}$ at $4^{\circ} \mathrm{C}$. Specific $\alpha$-BTX binding was inhibited by bringing the mixture to $0.05 \%$ (28.7 mM) $d$-tubocurarine. ${ }^{125} \mathrm{I}-\alpha$-BTX (New England Nuclear, Boston, MA) was added to a final concentration of $35 \mathrm{~nm}$ and 


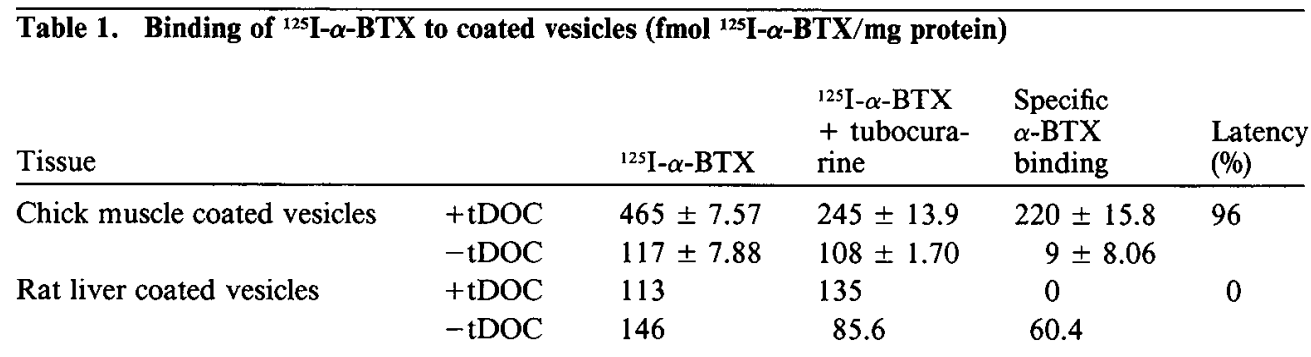

Bungarotoxin binding was measured according to the assay described in Materials and Methods. Values for chick muscle coated vesicles are expressed as mean \pm SD of duplicate values.

the solution incubated for $30 \mathrm{~min}$ at $20^{\circ} \mathrm{C}$. The coated vesicles or membrane fractions were then collected by centrifugation through $15 \%$ sucrose in $0.5 \%$ BSA at $170,000 \times g$ for $30 \mathrm{~min}$ in a Beckman airfuge. The pellets were gently washed with ice-cold MES buffer and radioactivity determined in an LKB $\gamma$-counter. Pellets were resuspended and protein determined by the fluorescamine method (Sims and Carnegie, 1975).

\section{Electron microscopy}

Coated vesicle samples were assessed for purity by elcctron microscopy of preparations negatively stained with $2 \%$ aqueous uranyl acetate. Density-shifted and unshifted coated vesicle fractions were removed from the gradient, adjusted to $2.5 \%$ glutaraldehyde, $\mathrm{pH} 6.5$, and fixed overnight at $4^{\circ} \mathrm{C}$. The vesicles were then pelleted by airfuge centrifugation at 23 p.s.i. for $30 \mathrm{~min}$, washed with three $15 \mathrm{~min}$ changes of $0.1 \mathrm{M}$ sodium cacodylate buffer, $\mathrm{pH} 7.3$, and postfixed in $1 \%$ osmium tetroxide in the same buffer for $1 \mathrm{hr}$ at $4^{\circ} \mathrm{C}$. Postfixed pellets were rinsed as above, dehydrated in graded ethanols through propylene oxide, and embedded in Araldite. Sections 500-600 $\AA$ were cut on an LKB-Ultratome-V ultramicrotome, stained with $2 \%$ uranyl acetate in $100 \%$ methanol- $70 \%$ ethanol and Reynolds lead citrate, and examined on a Philips 300 electron microscope.

\section{Density shift of AChE-containing coated vesicles}

Coated vesicles were incubated in a modified Karnovsky and Roots (1964) incubation medium (K-R) to produce a dense copper-iron-containing precipitate within the vesicles. In brief, $10 \mathrm{mg}$ acetylthiocholine iodide (ATCh) was dissolved in $6.4 \mathrm{ml} 0.1 \mathrm{M}$ sodium hydrogen maleate buffer, $\mathrm{pH} 6.5$. One $\mathrm{ml} 0.1 \mathrm{M}$ sodium citrate, $0.6 \mathrm{ml} 0.1 \mathrm{M}$ cupric sulfate, and $2 \mathrm{ml} 5 \mathrm{~mm}$ potassium ferricyanide were then added sequentially with vortexing between additions. Control solutions either contained $10^{-3} \mathrm{M}$ DFP or lacked the substrate ATCh. Coated vesicles in MES buffer were incubated with equal volumes of the reaction mixture for various periods at $4^{\circ} \mathrm{C}$. Discontinuous gradients were prepared in $5 \times$ $41 \mathrm{~mm}$ centrifuge tubes (Beckman). These gradients contained $0.08 \mathrm{ml}$ each of the following solutions: $100 \% \mathrm{D}_{2} \mathrm{O}-50 \%$ Ficoll $400-10 \%$ sucrose; $90 \% \mathrm{D}_{2} \mathrm{O}-30 \%$ Ficoll- $8 \%$ sucrose; $70 \% \mathrm{D}_{2} \mathrm{O}-23 \%$ Ficoll-6\% sucrose; $50 \% \mathrm{D}_{2} \mathrm{O}-16 \%$ Ficoll-5\% sucrose; $24 \% \mathrm{D}_{2} \mathrm{O}-10 \%$ Ficoll-4\% sucrose; and $9 \% \mathrm{D}_{2} \mathrm{O}-3 \%$ Ficoll-3\% sucrose in MES buffer. The incubation mixtures were layered on top and the tubes were centrifuged for $20 \mathrm{~min}$ at $60,000 \times g$ in a Beckman SW 50.1 rotor with adapters. Shifted and nonshifted coated vesicle-containing regions were removed from the tubes using Pasteur pipettes.

\section{Results}

\section{Chick embryo muscle coated vesicles contain latent AChRs}

When coated vesicles isolated from $17 \mathrm{~d}$ chick embryo muscle were assayed for the presence of AChRs, binding of ${ }^{125} \mathrm{I}-\alpha$-BTX, a toxin isolated from snake venom with a very high specific affinity for the nicotinic AChR (Chang and Lee, 1963), was present. Table 1 shows the results of a binding study performed on one such coated vesicle preparation. Specificity of the binding sites for ${ }^{125} I-\alpha$-BTX was determined by preincubating coated vesicles with $28.7 \mathrm{~mm}$ tubocurarine, a competitive inhibitor of $\alpha$-BTX binding, or a 100 -fold excess of unlabeled $\alpha$-BTX. The binding conditions (described in Materials and Methods) were similar to those used in previous studies (Devreotes and Fam- brough, 1975; Klett et al., 1973), and were found to saturate the ligand binding sites while minimizing the nonspecific interaction between the numerous positively charged residues of $\alpha$-BTX (Mebs et al., 1971) and clathrin, which carries a net negative charge (Rubenstein et al., 1981). Coated vesicles isolated from adult rat liver, an organ that contains no AChRs, had no specific ${ }^{125} \mathrm{I}-\alpha$-BTX binding sites. Table 1 also shows that $96 \%$ of the specific ${ }^{125} \mathrm{I}-\alpha$-BTX binding sites were latent, i.e., available for toxin binding only in the presence of detergent. These data are consistent with the hypothesis that coated vesicles contain AChRs oriented with their ligand binding sites facing the lumen of the vesicle, as would be expected if AChRs were being transported within the cell by coated vesicles. Scatchard (1949) analysis of the binding of $\alpha$-BTX to AChRs in chick muscle coated vesicles revealed a maximal bound ligand value of $716 \mathrm{fmol} / \mathrm{mg}$ protein and an association constant of $2.15 \times 10^{-10} \mathrm{M}$. These values are in agreement with previously published values (Heidmann and Changeux, 1978; Klett et al., 1973).

To confirm that the ${ }^{125} \mathrm{I}-\alpha$-BTX binding sites are associated with the coated vesicles and not with a contaminating structure, we employed agarose gel clcctrophorcsis as a final purification step (Rubenstein et al., 1981). Also, following incubation with ${ }^{125} \mathrm{I}-\alpha$-BTX, we used trypsin treatment to shift the electrophoretic mobility of coated vesicles as previously described (Schmid et al., 1982). Figure $1 A$ demonstrates that trypsin treatment of muscle coated vesicles increased their electrophoretic mobility on agarose gels, and that the position of the bound ${ }^{125} \mathrm{I}-\alpha-\mathrm{BTX}$ was shifted a commensurate amount. Pretreatment of the coated vesicles with $d$-tubocurarine before toxin binding eliminated all coated vesicle-associated ${ }^{125} \mathrm{I}-\alpha$-BTX seen on autoradiographs of the agarose gel (Fig. $1 B$ ).

This increase in mobility contrasted with results obtained using brain or liver coated vesicles in which an identical trypsin treatment decreased the mobility of the coated vesicles (Schmid et al., 1982). A possible explanation for these results may lie in the different protein degradation patterns effected by the trypsin treatment. We observed little cleavage of chick muscle clathrin by the trypsin concentration employed, while Schmid et al. (1982) reported an almost total cleavage of brain or liver clathrin to a $110 \mathrm{kDa}$ species. In contrast, total degradation of the 50-55 $\mathrm{kDa}$ polypeptides and light chains occurred with all 3 types of coated vesicles (data not shown).

As a further demonstration that the AChRs are associated with coated vesicles and not with smooth vesicle contaminants, we incubated coated vesicles in $0.5 \mathrm{~mm}$ Tris, $\mathrm{pH} 8.8$, to remove the clathrin coat. Figure $1 C$ shows that decoated vesicles migrated through the agarose gel much more slowly than did coated vesicles (Schmid et al., 1982), and formed a diffuse band near the origin. We also see in Figure $1 C$ that the bound ${ }^{125} \mathrm{I}-\alpha-\mathrm{BTX}$ associated with these vesicles was found in the same region near the origin of the gel.

In some experiments we observed a good deal of protein and ${ }^{125} \mathrm{I}-\alpha$-BTX at the gel origin. This was in contrast to results 
obtained with an identical preparation in which AChE and coated vesicle mobilities were compared (Benson et al., 1985, fig. $5)$. In the latter case, the vast majority of both $\mathrm{AChE}$ and coated vesicle proteins migrated through the gel. The most likely reason for this discrepancy is that in order to remove all unbound ${ }^{125} \mathrm{I}-$ $\alpha$-BTX, it was necessary to pellet the coated vesicles through sucrose, then wash and repellet before electrophoresis. Unfortunately, this treatment also produced coated vesicle aggregates that, owing to the sieving properties of the agarose gel, were unable to enter the gel and hence remained at the origin. A very careful resuspension was employed in the experiment shown in Figure $1 C$. After the initial isolation, the vesicles were allowed to incubate for several days at $4^{\circ} \mathrm{C}$ in MES buffer. This was followed by centrifugation for $5 \mathrm{~min}$ at $12,000 \times g$ and electrophoresis of the supernatant. As can be seen, very little material was found at the origin.

Figure $1 D$ demonstrates that liver coated vesicles showed very little association of ${ }^{125} \mathrm{I}-\alpha$-BTX with the coated vesicle-containing region when the incubation with toxin was carried out in the absence of detergent. This association was further decreased when detergent was present (see also Table 1). Presumably, the detergent eliminated nonspecific binding sites, and since no latent sites were exposed, total binding was reduced. Figure $1 D$ also shows that most of the ${ }^{125} \mathrm{I}-\alpha$-BTX that pelleted with the liver coated vesicles migrated towards the negative pole, as does free ${ }^{125} \mathrm{I}-\alpha$-BTX, owing to its positive charge at $\mathrm{pH} 6.5$.

Table 2 summarizes the enrichment of AChR (total, exposed, and latent) obtained in fractions taken during a typical coated vesicle purification from chick embryo muscle. In this case, we recovered $0.06 \%$ of the cell protein and $0.12 \%$ of the total AChRs in the purified coated vesicles. More important, we recovered $0.36 \%$ of the latent AChRs, but only $0.007 \%$ of the exposed AChRs, indicating a specific enrichment for sealed membrane vesicles carrying luminally oriented binding sites. We achieved a 5.54-fold enrichment of AChRs over the initial low-speed supernatant in purified coated vesicles, which is similar to the 3.17-fold enrichment in AChE achieved by Benson et al. (1985). Both are probably underestimates due to the high amount of clathrin-associated proteins in coated vesicles (Table 2).

As was mentioned in the introduction, Bursztajn and Fischbach (1984) recently presented evidence, based on in situ cytochemistry employing HRP coupled to $\alpha$-BTX, that chick embryo muscle coated vesicles contain AChRs. Their data, together with the biochemical evidence presented here, strongly indicate that chick embryo muscle coated vesicles contain AChRs. Since

Figure 1. Agarose gel electrophoresis of coated vesicles labeled with ${ }^{125} \mathrm{I}-\alpha$-BTX. Samples of coated vesicles were electrophoresed on $0.15 \%$ agarose gels as described by Rubenstein et al. (1981). The gel was partially dried by blotting, then stained with Coomassie blue to determine the location of the coated vesicles. In other preparations, it was determined that the Coomassie blue staining corresponds to the location of clathrin on the gel, as well as to the position of homogeneous coated vesicles, as shown by negative staining (Benson et al., 1985). After complete drying, the gel was autoradiographed for $24 \mathrm{hr}$ at $-70^{\circ} \mathrm{C}$, using a Cronex Lighting Plus intensification screen (Dupont) to determine the location of the ${ }^{125} \mathrm{I}-\alpha$-BTX. Arrows indicate gel origins. $A$, Chick embryo muscle coated vesicles $(50 \mu \mathrm{g})$ in MES buffer were first incubated with ${ }^{125} \mathrm{I}-\alpha-\mathrm{BTX}$ in the presence of tDOC, RNase, and BSA, then centrifuged, washed, and resuspended in $100 \mu \mathrm{l}$ MES buffer, as described for the binding assay in Materials and Methods. The sample was divided into 2 equal aliquots. To one was added trypsin ( $1 \% \mathrm{wt} / \mathrm{vol}$ final volume). No addition was made to the remaining aliquot. After $1 \mathrm{hr}$ at $37^{\circ} \mathrm{C}$, soybean trypsin inhibitor was added to all samples, which were then electrophoresed. $+T$, Trypsin-treated; $-T$, not trypsin-treated. $B$, A 50 $\mu \mathrm{g}$ aliquot of chick muscle coated vesicles was treated as in $A$, except that $28.7 \mathrm{~mm}$ tubocurarine was included in the ${ }^{125} \mathrm{I}-\alpha$-BTX incubation medium. $C$, A $50 \mu \mathrm{g}$ aliquot of chick muscle coated vesicles was in-
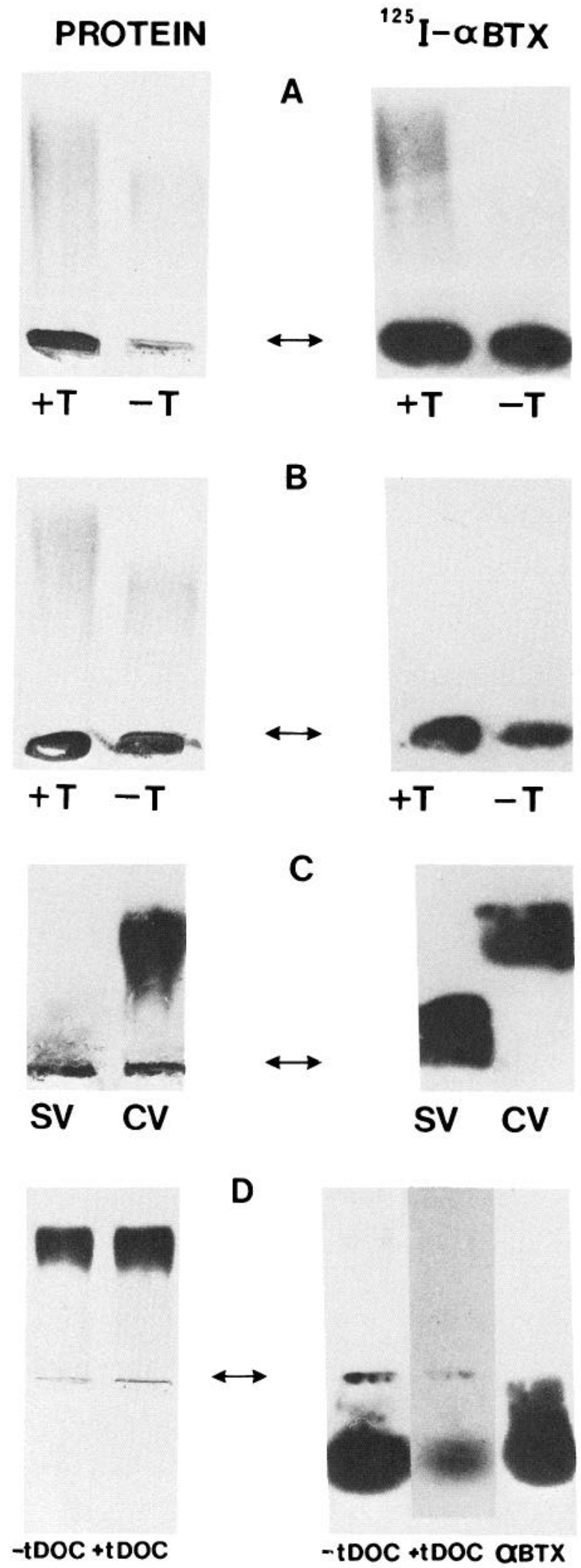

cubated with ${ }^{125} \mathrm{I}-\alpha$-BTX, as described in $A$, and divided into 2 aliquots. One was then incubated overnight in $0.5 \mathrm{M}$ Tris, $\mathrm{pH} 8.8$, and centrifuged at $35,000 \times \mathrm{g}$ for $30 \mathrm{~min}$, followed by resuspension in MES buffer. The other aliquot was untreated. $C V$, Coated vesicles; $S V$, uncoated vesicles. $D$, Two $25 \mu \mathrm{g}$ aliquots of rat liver coated vesicles were incubated in ${ }^{125} \mathrm{I}-$ $\alpha$-BTX as in $A$, except that tDOC was omitted from 1 aliquot. $+t D O C$, with $\mathrm{tDOC} ;-t D O C$, without tDOC. $\alpha B T X$, sample containing 10,000 cpm of ${ }^{125} \mathrm{I}-\alpha-\mathrm{BTX}$. 


\begin{tabular}{|c|c|c|c|c|}
\hline & $\begin{array}{l}\text { Low-speed } \\
\text { supernatant } \\
\text { (LSS) }\end{array}$ & $\begin{array}{l}\text { High-speed } \\
\text { pellet }\end{array}$ & $\begin{array}{l}\text { Ficoll-sucrose } \\
\text { supernatant }\end{array}$ & $\begin{array}{l}\text { Sucrose } \\
\text { gradient- } \\
\text { purified } \\
\text { coated } \\
\text { vesicles }\end{array}$ \\
\hline Protein $(\mathrm{mg})$ & 10,328 & 1352 & 185 & 6.44 \\
\hline Recovery (\%LSS) & - & 13.1 & 1.8 & 0.065 \\
\hline AchRs total (ng) $(+\mathrm{tDOC})$ & 7472 & 3206 & 275 & 9.1 \\
\hline Recovery (\%LSS) & - & 42.9 & 3.7 & 0.12 \\
\hline Enrichment (fold) & - & 3.2 & 2.05 & 1.96 \\
\hline AChRs exposed (ng) (-tDOC) & 5053 & 497 & 151 & 0.37 \\
\hline Recovery (\%LSS) & - & 9.8 & 3.0 & 0.007 \\
\hline Enrichment (fold) & - & 0.75 & 1.66 & 0.10 \\
\hline \multicolumn{5}{|l|}{ AChRs masked (ng) } \\
\hline (total - exposed) & 2419 & 2709 & 151 & 8.73 \\
\hline Recovery (\%LSS) & - & 112 & 6.2 & 0.36 \\
\hline Enrichment (fold) & - & 8.55 & 3.44 & 5.54 \\
\hline $\begin{array}{l}\text { Latency }(\%) \\
(\text { masked } / \text { total }) \times 100\end{array}$ & 32 & 84 & 45 & 96 \\
\hline
\end{tabular}

Successive stages in the coated vesicle purification were monitored for protein by the fluorescamine method (Sims and Carnegie, 1975) and for AChRs by the ${ }^{125}$ I- $\alpha$-BTX binding method described in Materials and Methods.

coated vesicles also contain AChE (Benson et al., 1985), an obvious question comes to mind: Does an individual coated vesicle carry both molecules, or are they segregated into different coated vesicles?

\section{AChE-mediated density shift of coated vesicles}

To address the aforementioned question, we have developed an AChE-mediated density shift method employing the widely used Karnovsky and Roots (1964) histochemical reaction for AChE. This reaction leads to the deposition of a very dense iron-copper complex that should render any closed membrane compartment containing AChE denser than those that contain no AChE.

Isolated chick embryo skeletal muscle coated vesicles were reacted with the K-R reagents for $16 \mathrm{hr}$ at $4^{\circ} \mathrm{C}$, with and without ATCh. The coated vesicles were then placed on Ficoll- $\mathrm{D}_{2} \mathrm{O}-$ sucrose step gradients and centrifuged as described in Materials and Methods. Figure 2 shows the result obtained. A very tight band appeared in the dense region of the gradient, which was not present when ATCh was not included. The inclusion of $10^{-4}$ $M$ DFP in the reaction mixture in the presence of ATCh also totally inhibited the generation of the new band (data not shown).

To determine whether this new band was generated because of the AChE-mediated density shift or through some artifactual aggregation, we monitored the presence of $\mathrm{AChE}$ in the 2 regions of the gradient by electron microscopy and enzymatic assay. Thin sections from pellets obtained from the nondensity-shifted portion of the gradient (Fig. 3) revealed cross sections of coated vesicles with light central regions identical to those seen in pellets from unreacted coated vesicles (Benson et al., 1985, fig. 1b). In contrast, the coated vesicle cross sections from density-shifted pellets appeared much darker, indicating that the deposition of the iron-copper complex had occurred. Some large membrane fragments containing no reaction product were also seen (Fig. 3 , arrowheads). Morphometric analysis of the density-shifted pellets from 3 preparations indicated that membrane fragments and other contaminating particles accounted for $8 \pm 2 \%$ of the particles. However, analysis of the starting material indicated that it contained $98 \pm 1.2 \%$ coated vesicles (Benson et al., 1985). Even if all the contaminating membrane had moved to the dense region of the gradient, it should have contributed only $2-4 \%$ of the total particles, while $80-90 \%$ of the sample shifted. Therefore, a reasonable explanation for this finding is that some of the AChE-containing coated vesicles actually exploded because of the accumulation of large amounts of iron-copper reaction product, followed by high-speed sedimentation. Consistent with this interpretation is the finding that if density-shifted coated vesicles were sedimented to form a pellet prior to fixation, either by longer centrifugation times during the density gradient centrifugation or after removal from the gradient, no intact coated vesicles were seen, but rather a heterogeneous mixture of membrane fragments and reaction product (data not shown). We have determined the time course for the density shift. It appeared to reach completion after a $4 \mathrm{hr}$ incubation with the K-R reagents. After this time, no further density shift of AChE or coated vesicles was observed.

We conducted a series of experiments in which we measured the AChE activity in shifted and unshifted coated vesicles using the Johnson and Russell method (1975), and measured protein by the fluorescamine (Sims and Carnegie, 1975) or the Bradford (1976) method. These experiments were designed to address the concern that $\mathrm{AChE}$ adherent to the outside of coated vesicles, soluble in the reaction mixture, or within other vesicles might cause a nonspecific shift of coated vesicles. Incubation with the $\mathrm{K}-\mathrm{R}$ reagents with or without ATCh does not affect AChE activity as measured by the Johnson and Russell (1975) method (data not shown). In Figure 4, we show that, in untreated muscle coated vesicles, $74 \%$ of the $\mathrm{AChE}$ and $69 \%$ of the protein in the sample shifted to a greater density when incubated with K-R reagents and ATCh. Solubilization of the coated vesicle membrane with Triton X-100 did not inhibit the activity of the AChE (data not shown), but prevented the density shift of coated vesicles such that only $7 \%$ of the AChE and $3 \%$ of the protein were found in the lower portion of the gradient. Preincubation of the coated vesicles with DFP to inactivate all endogenous AChE, followed by addition of eel electric organ $\mathrm{AChE}$ to restore the level of AChE activity, led to a shift of only $5 \%$ of the AChE and $7 \%$ of the protein. Thus, external AChE, although reacting with the K-R reagents and causing a precipitate to form in the reaction tube, did not cause a change in the density of the coated vesicles. Conversely, preincubation of the coated vesicles with 


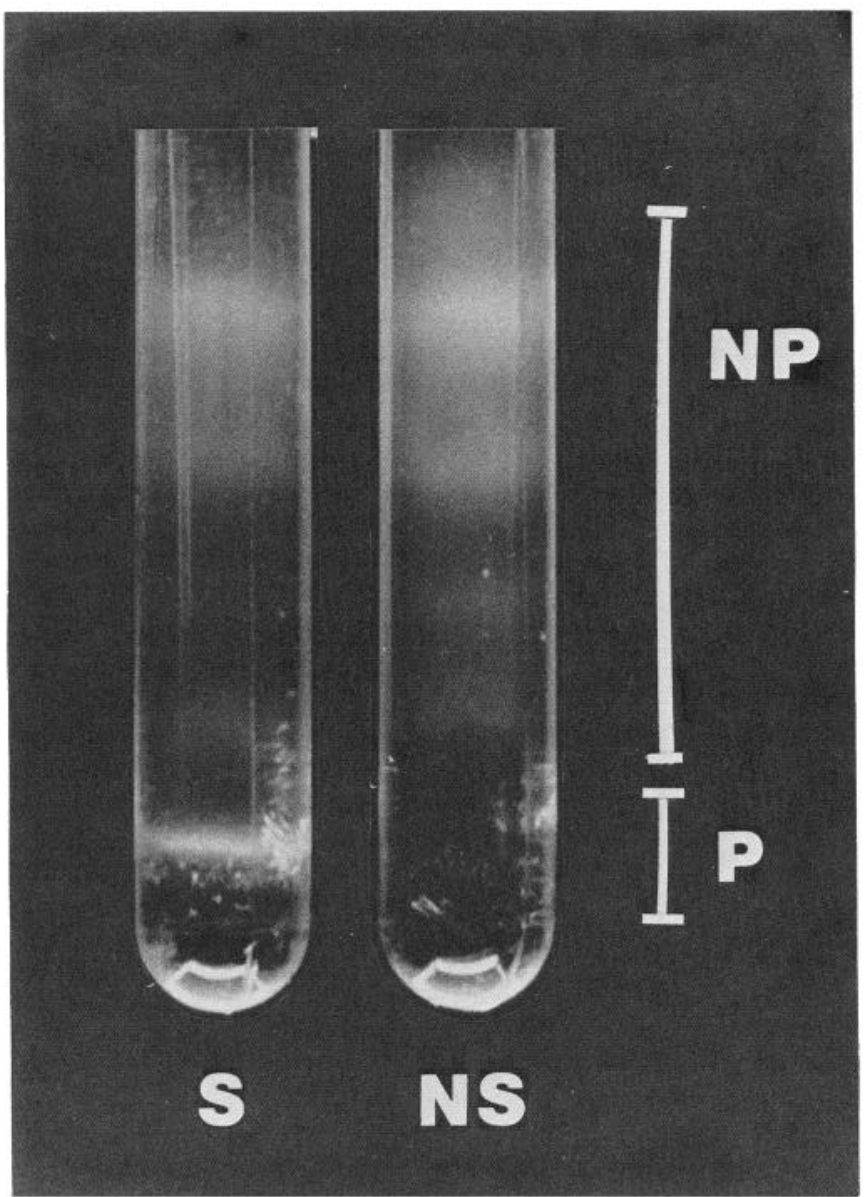

Figure 2. Density shift of AChE-containing coated vesicles. Coated vesicles $(100 \mu \mathrm{g})$ were incubated overnight in Karnovsky and Roots (1964) staining medium, $\mathrm{pH} 6.5$, in the presence $(S)$ or absence $(N S)$ of $1 \mathrm{mg} / \mathrm{ml} \mathrm{ATCh}$, as described in Materials and Methods. $N P$ and $P$, Density-shifted and unshifted coated vesicle-containing regions, respectively, from Ficoll-sucrose- $\mathrm{D}_{2} \mathrm{O}$ gradients.

echothiopate, a hydrophilic, irreversible esterase inhibitor that acts quickly on exposed esterase but diffuses slowly across membranes (Rotundo and Fambrough, 1980b), did not significantly decrease the density shift of coated vesicles, with $69 \%$ of the $\mathrm{AChE}$ and $67 \%$ of the protein shifting to the lower portion of the gradient. We conclude that functional AChE must be contained within coated vesicles and that the iron-copper precipitate is formed within these coated vesicles, changing their mobility on density gradients.

We compared the AChE activity, protein, and AChR number found in density-shifted and unshifted coated vesicles. As is shown in Table 3,90\% of the AChE activity in coated vesicles from this preparation shifted when ATCh was present, while with no substrate present only $6 \%$ shifted. Of the total coated vesicle protein, $86 \%$ shifted in the presence of ATCh, while less than $2 \%$ shifted when no substrate was present. We assayed ${ }^{125} \mathrm{I}-$ $\alpha$-BTX binding activity as a marker for AChRs in the shifted and unshifted portions of the gradients. Table 3 shows that $89 \%$ of the AChRs also density shifted in concert with the AChE. These results were similar to those obtained in other preparations of muscle coated vesicles (data not shown).

From these findings, we can draw 2 reasonable conclusions. One is that at least some coated vesicles that density shift contain both AChE and AChR molecules. Second, most of the coated vesicles in our isolated coated vesicle population contain at least one and possibly more AChE molecules.

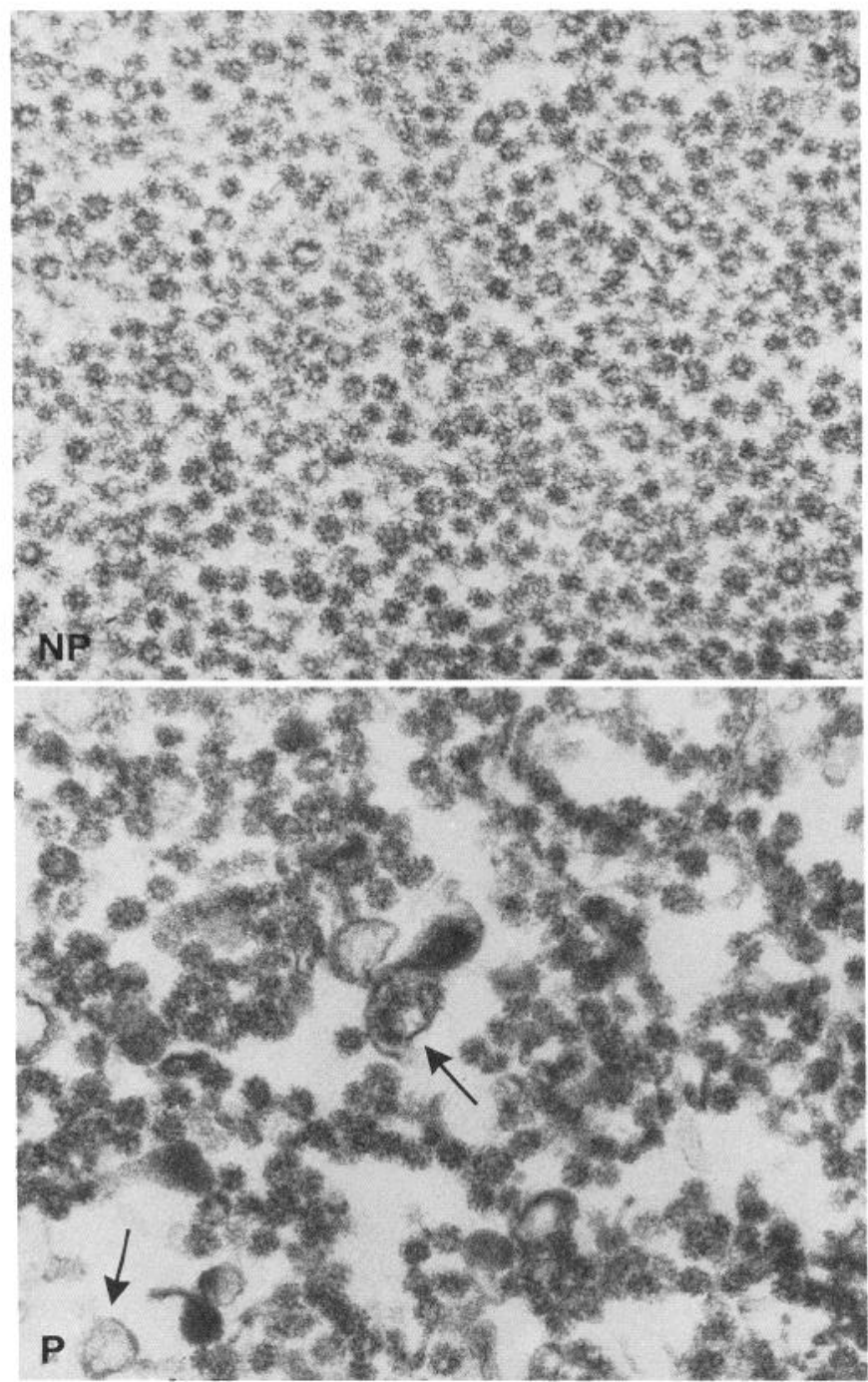

Figure 3. Electron microscopy of shifted and unshifted coated vesicles. Vesicles from the upper $(N P)$ and lower $(P)$ regions of the gradient described in Figure $2(S)$ were fixed overnight in $2.5 \%$ glutaraldehyde and pelleted for $60 \mathrm{~min}$ at $140,000 \times \mathrm{g}$. Embedding, sectioning, and staining were as described in Materials and Methods. Magnification, $51,300 \times$.

\section{$A C h E$-mediated density shift of coated vesicles isolated from DFP-treated and recovered myotubes}

Since we do not know the origin and destination of the AChEcontaining coated vesicles isolated from whole muscle, we isolated coated vesicles from cultures of myotubes that had been DFP-treated and allowed to recover for $2 \frac{1}{2} \mathrm{hr}$, as previously described (Benson et al., 1985). These coated vesicles contained latent AChE activity and also AChRs, as was indicated by specific binding sites for ${ }^{125} \mathrm{I}-\alpha$-BTX; see Table 4 . The 2.3-fold enrichment of AChRs shown in Table 4 is, again, similar to that seen with coated vesicles isolated from whole muscle (1.96-fold), and is also similar to the 2.4-fold enrichment in AChE in coated vesicles isolated from myotubes (Benson et al., 1985; table V).

We performed density shift experiments with the coated vesicles isolated from DFP-treated and recovered cells under conditions identical to those described in the previous section. Again, we generated a denser, coated vesicle band that was not produced either when ATCh was omitted or $10^{-4} \mathrm{M}$ DFP was present during incubation with K-R reagents (data not shown).

These experiments were carried out on coated vesicles isolated 


\begin{tabular}{|c|c|c|c|c|}
\hline $\begin{array}{l}\text { ATCh } \\
(+ \text { or }-)\end{array}$ & $\begin{array}{l}\text { Gradient } \\
\text { region } \\
\text { (P or } \\
\mathrm{NP})\end{array}$ & $\begin{array}{l}\text { AChE } \\
\text { activity } \\
\text { (cpm) }\end{array}$ & $\begin{array}{l}\text { Total } \\
\text { protein } \\
(\mu \mathrm{g})\end{array}$ & $\begin{array}{l}\text { Specific } \\
\alpha \text {-BTX } \\
\text { binding } \\
\text { (fmol) }\end{array}$ \\
\hline+ & NP & $4315(10 \%)$ & $8(14 \%)$ & $1.7(11 \%)$ \\
\hline+ & $\mathbf{P}$ & $37,918(90 \%)$ & $48(86 \%)$ & $13.7(89 \%)$ \\
\hline- & NP & $32,622(94 \%)$ & $84(100 \%)$ & $17.5(100 \%)$ \\
\hline- & $\mathbf{P}$ & $2273(6 \%)$ & 2 & 0.04 \\
\hline
\end{tabular}

Coated vesicles from embryonic chick skeletal muscle were incubated in KamovskyRoots staining medium and density-shifted as described in Materials and Methods. The gradient regions $\mathrm{P}$ and NP correspond to the density-shifted and unshifted coated vesicles, respectively, as shown in Figure 2. Protein, AChE, and ${ }^{125} \mathrm{I}-\alpha-\mathrm{BTX}$ binding studies were performed as described in Materials and Methods.

from 3 different preparations of cultured cells. (Two preparations were done in duplicate.) Table 5 summarizes the results when $\mathrm{AChE}, \mathrm{AChR}$, and protein assays were performed on the density-shifted coated vesicles. We were unable to density shift as much of the AChE as we could when using coated vesicles isolated from whole muscle (Table 3).

The amounts of AChE, AChR, and protein shifted were different in each preparation. However, the values varied in a similar manner. We compared the percentages of $\mathrm{AChE}$ and AChRs shifted in each of the 5 determinations shown in Table 5 , and found, by a chi-square analysis, an almost direct proportionality between the 2 variables. We also calculated a correlation coefficient of 0.95 for the variation between the percentages of AChE and AChRs density-shifted using the Pearson method, which describes the relationship between 2 mutually dependent variables. The shift of total protein was also shown by similar plots to be proportional to the shifted AChRs and AChE (data not shown). Therefore, we feel reasonably confident in extrapolating from these data: If we shifted $90 \%$ of the AChE, an amount similar to that shifted when coated vesicles from whole muscle were employed (Table 3), we would expect that $85 \%$ of the AChRs and $76 \%$ of the protein would shift as well. These hypothetical results are quite similar to those actually obtained with coated vesicles isolated from whole muscle (Table 3 ). Since they carry newly synthesized AChE (Benson et al., 1985), coated vesicles from these muscle cultures are from the exocytotic pathway. The results here suggest, therefore, that most of the coated vesicles isolated from whole muscle are likely to be exocytotic as well.

\section{Discussion}

Our data are consistent with the hypothesis that AChRs are true components of coated vesicles isolated from both chick embryo

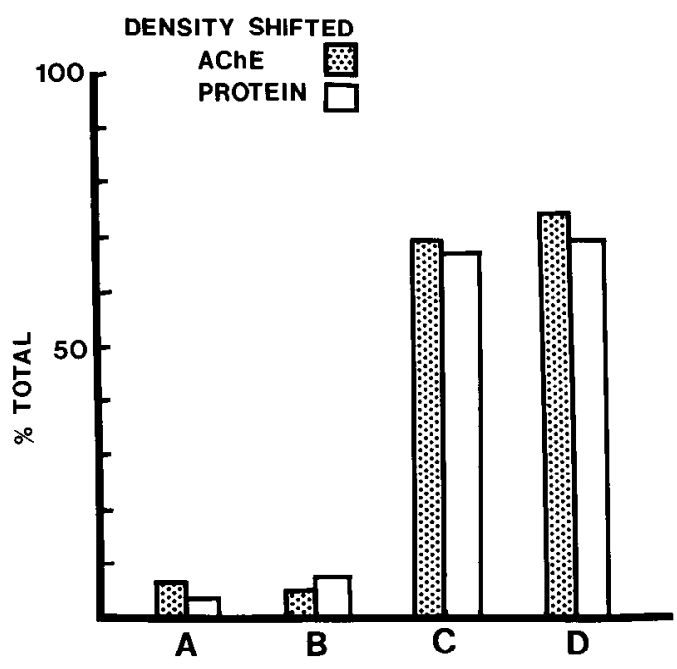

Figure 4. Effect of AChE inhibitors and exogenous AChE on the density shift of coated vesicles. Chick muscle coated vesicles, $200 \mu \mathrm{g}$, were purified on a Ficoll-sucrose gradient and divided into 4 equal aliquots designated $A, B, C$, and $D$. Aliquot $B$ was brought to $10^{-5} \mathrm{M}$ DFP and aliquot $C$ to $10^{-5} \mathrm{M}$ echothiopate. After $10 \mathrm{~min}$ at $22^{\circ} \mathrm{C}$, all 4 aliquots were pelleted in a Beckman airfuge, and the resulting pellets were washed 3 times and resuspended with MES buffer. Aliquot $A$ was then brought to $0.5 \%$ Triton X-100, and sufficient AChE from electric eel was added to aliquot $B$ to yield a rate of hydrolysis of $A C h$ equivalent to that of $50 \mu \mathrm{g}$ of untreated coated vesicles. Aliquot $D$ was untreated. The K-R reagents were added to all aliquots and, after $16 \mathrm{hr}$ at $4^{\circ} \mathrm{C}$, density gradient centrifugation was performed as decribed in Materials and Methods. The upper and lower portions of cach gradicnt wcre assaycd for protein (clear bars) and AChE (dotted bars). Only the percentages of $\mathrm{AChE}$ and protein found in the lower gradient region are shown.

skeletal muscles and cultured chick embryo myotubes. We think it unlikely that the AChRs are contained in a vesicle or other organelle that consistently purifies with coated vesicles through multiplc purification steps, including agarose gel electrophoresis under various conditions, though we cannot exclude this possibility. Bursztajn and Fischbach (1984) have also concluded that AChRs are contained in chick muscle coated vesicles based on the cytochemical localization of HRP- $\alpha$-BTX in cultured chick embryo myotubes.

We can calculate the approximate number of AChRs in our isolated coated vesicles from Scatchard (1949) analysis of binding studies. Assuming a molecular weight of 45,000,000 for a coated vesicle (Stevens et al., 1983) and 2 ligand binding sites per AChR (Chang and Lee, 1963; Heidmann and Changeux, 1978), we calculate that there is an AChR molecule present in approximately 1 in 60 coated vesicles. This is in contrast to our

Table 4. Protein and AChR number and enrichment at successive stages in coated vesicle purification

from cultured chick embryo myotubes

\begin{tabular}{|c|c|c|c|c|}
\hline & $\begin{array}{l}\text { Low-speed } \\
\text { supernatant } \\
\text { (LSS) }\end{array}$ & $\begin{array}{l}\text { High-speed } \\
\text { pellet }\end{array}$ & $\begin{array}{l}\text { Ficoll-sucrose } \\
\text { supernatant }\end{array}$ & $\begin{array}{l}\text { Sucrose } \\
\text { gradient- } \\
\text { purified } \\
\text { coated } \\
\text { vesicles }\end{array}$ \\
\hline Protein (mg) & 240.5 & 151.2 & 15.8 & 1.12 \\
\hline Recovery (\%LSS) & - & 62.9 & 6.6 & 0.47 \\
\hline AChRs (ng) & 313.2 & 72.5 & 25.6 & 3.4 \\
\hline Recovery (\%LSS) & - & 23.1 & 8.16 & 1.08 \\
\hline Enrichment (fold) & - & 0.37 & 1.25 & 2.3 \\
\hline
\end{tabular}

Protein and AChR were determined as described in Materials and Methods. 


$\begin{aligned} & \text { Table 5. Percentages of AChE, AChKs, and protein density-shifted } \\
& \text { using coated vesicles isolated from DFP-treated and recovered } \\
& \text { myotubes }\end{aligned}$
\begin{tabular}{llll} 
Preparation & AChE & AChRs & Protein \\
\hline 1 & 54 & 46 & 47 \\
2a & 36.2 & 38.2 & 46.3 \\
2b & 38.7 & 38.4 & 33.3 \\
3a & 63 & 63 & 55 \\
3b & 73 & 75 & 50 \\
Control & 5 & 0.04 & 2
\end{tabular}

Three preparations of coatcd vesicles were purificd from DFP-treated cultured chick embryo myotubes allowed to synthesize AChE for $2 \frac{1 / 2}{\mathrm{hr}}$, as described in Materials and Methods. Density shift experiments and assays for protein, AChE and AChRs were performed as described. Duplicate experiments were performed with coated vesicles from preparations 2 and 3 . An aliquot of the coated vesicles from each preparation was incubated in the absence of ATCh as a control. The control of preparation 1 is shown, but all controls were essentially identical. The displayed values represent the percentage of AChE activity, specific ${ }^{125} I-\alpha-B T X$ binding, and total protein found in the lower third of the gradient.

density shift results, which indicate that $70-90 \%$ of the coated vesicles contain at least 1 molecule of AChE. Rotundo and Fambrough (1982) have calculated that there are about 100 times as many newly synthesized AChE molecules in transit to the cell surface at any one time as there are AChR molecules, which is consistent with our data. Although Bursztajn and Fischbach (1984) have estimated that about $40 \%$ of coated vesicles found underlying "hot spots" on the myotube plasma membrane carry an $\mathrm{AChR}$, this estimate is considerably higher than the percentage of the total cell coated vesicle population carrying AChRs, since the regions underlying "hot spots" are likely to be enriched in coated vesicles carrying AChRs compared to other areas of the cytoplasm.

The density shift experiments on chick embryo skeletal muscle indicate that when the AChE-containing coated vesicles shift their density, an approximately equivalent percentage of AChRs and a slightly smaller amount of the total protein shift with them. We conclude that there is a population of coated vesicles that is simultaneously involved in transporting an integral membrane protein, the $\mathrm{AChR}$, and a secretory protein, the $\mathrm{AChE}$, within the cell. Since we have not been able to attain a $100 \%$ shift of the AChE, we do not know if there are any vesicles that contain AChR but not AChE. However, from the calculations outlined above, we know that many coated vesicles contain $\mathrm{AChE}$ but do not contain an $\mathrm{AChR}$. We interpret this to mean that vesicles are not obligated to transport both proteins together. We suspect that the number of vesicles containing both proteins is simply a function of the amount of each protein available to be transported and the number of coated vesicles available at the appropriate transport steps.

Our density shift experiments on coated vesicles isolated from DFP-treated and recovered myotubes suggest that over $75 \%$ of this population contain newly synthesized, soluble AChE. Similar results are obtained with coated vesicles isolated from whole muscle. We have shown that this is not an artifactual shift produced by aggregation or external precipitate formation (Fig. 4 ). One possible explanation for the finding that a large proportion of coated vesicles are in the exocytotic pathway is that our isolation may select for exocytotic coated vesicles, possibly because of their small size as compared to endocytotic coated vesicles (Friend and Farquhar, 1967). Inconsistent with this interpretation are data recently reported by Helmy ct al. (1986). These investigators employed the AChE-mediated density shift methodology to isolate significantly enriched populations of either endocytotic or exocytotic coated vesicles from perfused rat liver, using an isolation technique essentially identical to that described here (Pilch et al., 1983). In contrast to the results employing muscle coated vesicles, fewer than $30 \%$ of the coated vesicles in rat liver appear to be transporting esterase exocytotically, while over $50 \%$ appear to be endocytotic (Helmy et al., 1986).

A second explanation, which we favor, is that a high percentage of the total coated vesicles in developing muscle cells are indeed exocytotic. Developing muscle cells in vivo and in vitro are rapidly increasing in volume, and hence their surface area is also increasing. Thus, one would predict that much more surface membranc is bcing added than removed during this time. Bursztajn and Fischbach (1984) have obtained results consistent with this prediction. In mixed chick embryo skeletal muscle-fibroblast cultures incubated with HRP for $1 \mathrm{hr}$ and then treated with diaminobenzidine to localize HRP, the fibroblasts are filled with HRP-containing vesicles, while the myotubes contain very few. This demonstrates that myotubes are very inactive in endocytosis, as might be expected for a cell that is rapidly expanding in surface area. Although it remains to be seen whether coated vesicles transport membrane to the cell surface, they may contribute to the total membrane being added to, or removed from, the plasma membrane. In this case, one would expect the results we find: A high percentage of the coated vesicles in the growing myotube is engaged in exocytosis, while a much smaller percentage is engaged in endocytosis.

The density shift experiments from DFP-treated and recovered cells indicate that the majority of isolated coated vesicles that contain an AChR also contain at least one newly synthesized AChE molecule. We cannot definitively prove that the $\mathrm{AChR}$ molecules are newly synthesized as well; however, this seems a reasonable hypothesis. Devreotes and Fambrough (1975) have demonstrated that surface AChRs are not recycled in embryonic chick skeletal muscle cells, as are many classes of receptors in other cell types, but are, instead, internalized at a slow rate and then degraded. It is unlikely that a newly synthesized AChE molecule can share a common transport vesicle with an internalized AChR destined for degradation. The data of Bursztajn and Fischbach (1984) also suggest that most AChRs in coated vesicles are exocytotic.

The AChE-mediated density shift technique-which should be employable with other cholinesterases as well-combined with the ability to inactivate all preexisting AChE with DFP, offers several advantages as a means of isolating subcellular organelles, including coated vesicles, that are involved in exocytotic membrane processes in cells that synthesize and secrete AChE. In addition to skeletal muscle cells, certain cholinergic neurons, as well as cells and tissues innervated by these neurons, synthesize and secrete AChE, making them potentially amenable to this density shift technology. Therefore, one may be able to demonstrate the cotransport of AChE and any other secretory or integral plasma membrane proteins for which one has an antibody or other probe, in the same population of exocytotic coated vesicles, or possibly in other cellular organelles along the secretory pathway. The shifted esterase-containing organelles can be followed biochemically (Johnson and Russell, 1975) and morphologically (Karnovsky and Roots, 1964; and see Fig. 3). Because of the extremely high turnover number of $\mathrm{AChE}-10^{7} \mathrm{ACh}$ molecules/AChE active site/hr (Vigny et al., 1978)-it is possible that the presence of a single active AChE molecule would result in the deposition of enough iron-copper complex to produce a density shift.

The availability and low cost of purified eel electric organ AChE makes density shift technology useful in investigating the cndocytotic pathway as well. The grcat stability of the AChE should allow it to be coupled to various molecules while retaining its activity. In this regard, in collaboration with Dr. A. Schwartz, we have recently chemically added galactose residues to eel $\mathrm{AChE}$ with no loss of enzymatic activity, and have dem- 
onstrated that it becomes a high-affinity ligand for the hepatocyte asialoglycoprotein receptor (Helmy et al., 1986). By treating a perfused rat liver with DFP and, immediately after a short washout, coperfusing galactose-coupled $\mathrm{AChE}$ and ${ }^{125} \mathrm{I}$-insulin for $3 \mathrm{~min}$ through the portal vein, we have shown that both ligands are rapidly co-endocytosed into coated vesicles (Helmy et al., 1986), which can be density-shifted. We are currently using this technique to isolate other organelles in the endocytotic pathway (e.g., the endosome).

One problem with the density shift technique is that we cannot quantitatively shift all of the active AChE in our population. From coated vesicle preparation to preparation, we see variable amounts shifted, from 40 to over $90 \%$. We do not know the precise reason for this variability. It may depend on the permeability of the individual vesicles, which may vary considerably. Another possibility is that some of the AChE may fail to cleave ATCh, causing no precipitate to form, while successfully cleaving $\mathrm{ACh}$, so that it is measured by the Johnson and Russell (1975) assay.

The second problem with the technique is that the accumulation of the reaction product is likely to render the membrane of the coated vesicle (or any other organelle) quite fragile, analogous to a balloon filled with lead pellets. Subsequent centrifugations or other procedures such as vortexing or vigorous pipetting are likely to rupture the membrane. Also, any iontransport activity may be damaged by the high concentration of the iron-copper complex. We should point out that both AChE activity and the ability of AChRs to specifically bind ${ }^{125}$ I$\alpha$-BTX survive the reaction and sedimentation. We hope other membrane-associated activities (e.g., ligand-receptor interactions and enzymatic activities) will also survive.

While the AChE-mediated density shift technique is applicable to the study of intracellular transport in many tissues, using AChE as a tag, we believe that the true power of the method will be revealed in studies of the nervous system. The cholinergic pathways of the CNS have been shown to be very important in certain neurological diseases. The AChE-mediated density shift technique offers a possible means for examining the synthesis and transport of molecules of the cholinergic system.

\section{References}

Benson, R. J. J., K. Porter-Jordan, P. Buoniconti, and R. E. Fine (1985) Biochemical and cytochemical evidence indicates that coated vesicles in chick embryo myotubes contain newly synthesized acetylcholinesterase. J. Cell Biol. 101: 1930-1940.

Bradford, M. (1976) A rapid and sensitive method for the quantitation of microgram quantities of protein utilizing the principle of proteindye binding. Anal. Biochem. 72: 248-254.

Bursztajn, S., and G. D. Fischbach (1984) Evidence that coated vesicles transport acetylcholine receptors to the surface membrane of chick myotubes. J. Cell Biol. 98: 498-506.

Chang, C. C., and C. Y. Lee (1963) Isolation of neurotoxins from the venom of Bungarus multicinctus and their modes of neuromuscular blocking action. Arch. Int. Pharmodyn. Ther. 144: 241-257.
Devreotes, P., and D. Fambrough (1975) Acetylcholine receptor turnover in membranes of developing muscle fibers. J. Cell Biol. 65: 335358.

Friend, D. G., and M. G. Farquhar (1967) Functions of coated vesicles during protein absorption in the rat vas deferens. J. Cell Biol. 35: $357-376$

Heidmann, T., and J. P. Changeux (1978) Structural and functional properties of the acetylcholine receptor protein in its purified and membrane bound states. Annu. Rev. Biochem. 47: 317-357.

Helmy, S., K. Porter-Jordan, E. A. Dawidowicz, P. Pilch, A. L. Schwartz, and R. E. Fine (1986) Separation of endocytic from exocytic coated vesicles using a novel cholinesterase mediated density shift technique. Cell 44: 497-506.

Johnson, C. D., and R. L. Russell (1975) A rapid simple radiometric assay for cholinesterase, suitable for multiple determinations. Anal. Biochem. 64: 229-238.

Karnovsky, M. J., and L. Roots (1964) A direct coloring method for cholinesterases. J. Histochem. Cylochem. 12: 219-221.

Klett, R. P., B. W. Fulpius, D. Cooper, M. Smith, E. Reich, and L. D. Possani (1973) The acetylcholine receptor. J. Biol. Chem. 248: 68416853.

Lowry, O. H., N. J. Rosebrough, A. L. Farr, and R. J. Randall (1951) Protein measurement with the Folin phenol reagent. J. Biol. Chem. 193: 265-275.

Mebs, D., K. Narita, S. Iwanaga, Y. Samejima, and C. Y. Lee (1971) Amino-acid sequence of BTX from the venom of Bungarus multicinctus. Biochem. Biophys. Res. Commun. 72: 479-488.

Pilch, P., M. Shia, R. Benson, and R. Fine (1983) Coated vesicles participate in the receptor-mediated endocytosis of insulin. J. Cell Biol. 93: 133-138.

Porter-Jordan, K., R. J. J. Benson, and R. E. Fine (1982) Localization of the acetylcholine receptor and acetylcholinesterase in coated vesicles. J. Cell Biol. 95: 405a.

Rotundo, R. L., and D. M. Fambrough (1980a) Secretion of acetylcholinesterase: Relation to acetylcholine receptor metabolism. Cell 22: 595-602.

Rotundo, R. L., and D. M. Fambrough (1980b) Synthesis, transport and fate of acetylcholinesterase in cultured chick embryo muscle cells. Cell 22: 583-594.

Rotundo, R., and D. Fambrough (1982) Synthesis, transport and fate of acetylcholinesterase and acetylcholine receptors in cultured muscle. In Membranes in Growth and Development, J. Hoffman, G. Giebish and L. Bolis, eds., pp. 259-286, Alan R. Liss, New York.

Rubenstein, J. L. R., R. E. Fine, B. D. Luskey, and J. E. Rothman (1981) Purification of coated vesicles by agarose gel electrophoresis. J. Cell Biol. 89: 357-361.

Scatchard, G. (1949) The attraction of proteins for small molecules and ions. Ann. NY Acad. Sci 51: 660-672.

Schmid, S., A. Matsumoto, and J. E. Rothman (1982) A domain of clathrin that forms coats. Proc. Natl. Acad. Sci. USA 79: 91-95.

Sims, N. R., and P. R. Carnegie (1975) Use of fluorescamine for the estimation of protein in the presence of the detergent Triton X-100. Anal. Biochem. 65: 578-589.

Stevens, A. C., J. F. Hainfeld, J. S. Wall, and C. J. Steer (1983) Mass distributions of coated vesicles isolated from liver and brain: Analysis by scanning electron microscopy. J. Cell Biol. 97: 1714-1723.

Vigny, M., S. Bon, J. Massoulie, and F. Leterrier (1978) Active site catalytic efficiency of acetylcholinesterase molecular forms in Electrophorus, torpedo, rat and chicken. Eur. J. Biochem. 85: 317-323. 